\title{
Habilidades sociales en la práctica docente: una mirada desde los actores de la educación básica
}

\section{Social skills in teaching practice: a look from the actors of basic education}

Laura Beatriz Fernández Delgado ${ }^{1}$

\section{Resumen}

Hoy en día los principios de calidad que sustentan los planes de estudios en Educación Básica son la inclusióny la equidad, así mismo se enfatiza el manejo de situaciones para la convivencia, cultura de paz, valores, actitudes y comportamientos que prevengan la violencia y solucionen conflictos. Sin embargo, la complejidad de la práctica educativa es enorme y muchas veces la práctica del quehacer docente deja de lado el aspecto humano del mismo sin centrarse en su persona, por lo que es necesario indagar si posee las habilidades sociales necesarias para su rol como agente de cambio hacia nuevas formas de saber, hacer y ser. El objetivo del estudio es analizar desde la mirada de docentes, directores y practicantesel desarrollo de dos Habilidades Sociales: Asertividad e Inteligencia emocional, revelar roles de trabajo y determinar áreas de oportunidad. Se realizó en dos preescolares y tres primarias. La orientación metodológica es el estudio de caso, enfoque cualitativo y alcance descriptivo. Las técnicas utilizadas fueron guía de entrevista y notas de campo. Los datos se analizaron por medio de la triangulación de respuestas y las tendencias se muestran en gráficas. Se encontró que existe una gran necesidad de trabajar creencias pedagógicas de los docentes ya que persisten aspectos de modelos educativos tradicionales en el aula, los actores educativos demandan capacitación presencial o mixta y no solo virtual en lo que refiere a la sensibilidad humana para aplicar las habilidades sociales de acuerdo a las metas de las tendencias actuales en educación.

1 Laura Beatriz Fernández Delgado. Académica de tiempo completo en la Universidad Pedagógica Nacional del Estado de Chihuahua, Campus Camargo, México. Es licenciada en Psicología y maestra en Psicología Aplicada a la Educación. Correo electrónico: lfernandez@upnech.edu.mx

ID: https://orcid.org/0000-0002-9842-3110 
RECIE. Revista Electrónica Científica de Investigación Educativa Vol. 4, núm. 2, enero-diciembre 2019, pp. 1303-1315.

\title{
Palabras clave
}

Habilidades sociales, educación básica, profesores.

\begin{abstract}
Nowadays, the quality principles that sustain basic education study plans are inclusion and equity, as well as handling of situations for coexistence, peace culture, values, attitudes and behaviors that prevent violence and solve conflicts are emphasized. However, the complexity of the educational practice is enormous and often, the practice of teaching leave aside the human aspect of it, without focusing on their person, so it is necessary to investigate If the teachers have the necessary social skills for their role as change agent toward new ways of knowing, doing and being. The study objective is analyze from teachers perspective, directors and practitioners the development of two social skills: assertiveness and emotional intelligence, to reveal work roles and determine opportunity areas. The investigation was realized in two preschools and three elementary school. The methodological orientation is the case study, with a qualitative approach and a descriptive scope. The techniques used are interview guide and field written notes. The data is analyzed through the triangulation of responses and the trends are shown by means of graphs. Wewerefoundthatthereis a great need to work pedagogical teachers beliefs, due to there are being traditional aspects of educational models persisting in the classroom, the educational aspects demand face-to-face or joint schooling and not only virtual trainingwith regard to human sensitivity and apply the social skills according to the goals of current trends in education.
\end{abstract}

\section{Keywords}

Social skills, basic education, teachers.

\section{Introducción}

Hoy en día los principios de calidad que sustentan los planes de estudios en Educación Básica (EB)son la inclusióny la equidad, por lo que una de las estrategias de la Secretaria de Educación Pública por medio del Plan de Estudios 2011 es promover las competencias para la vida quea su vez enfatizan el manejo de situaciones para la convivencia y para la vida en sociedad, entre otras.Por otro lado, el Nuevo Modelo Educativo propone los aprendizajes clave para la educación integral con los que busca conocimientos, prácticas, habilidades, actitudes y valores fundamentales que contribuyen sustancialmente al crecimiento integral del estudiante y 
posibiliten que la persona desarrolle un proyecto de vida y disminuya el riesgo de que sea excluida socialmente (Secretaria de Educación Publica, 2017).

Así mismo, la UNESCOen 1999enfatizóconstruir juntos una Cultura de la Paz, por medio de una serie de valores, actitudes y comportamientos que previenen la violencia y los conflictos desde sus causas con diálogo entre las personas, los grupos y las naciones. Dos de sus ámbitos de acción son promover esta cultura por medio de la educación y promover la comprensión, la tolerancia y la solidaridad.

\section{Planteamiento del problema}

Partiendo de la idea de la complejidad de la práctica educativa, vale la pena repensar que los planes y programas con enfoques por competencias que tienen su origen en propuestas de países de la OCDE, requieren una disposición y compromiso de los docentes para llegar a las metas establecidas. Bautista Martínez citado por Gimeno (2008)en el capítulo III de su obra Educar por competencias, ¿qué hay de nuevo?, refiere a una de lascompetencias más importantes en la actualidad, las necesarias para la ciudadanía, menciona "El agente pedagógico que es el profesor, cuando ejerce como tal, es un serhumano que actúa y ese papel no puede entenderse al margen de la condición humana, por muy tecnificado que se quiera sea ese oficio" (p.103).

En este orden de ideas, las Habilidades Sociales HHSS con las que cuente el profesor serán esenciales, ya que sin ellas le será muy difícil promover la evolución hacia nuevas formas de saber, de hacer y de ser. Ante este panorama, uno de los mayores desafíos empieza con un gran esfuerzo para abrir caminos hacia el descubrimiento de necesidades o áreas de oportunidad donde se puedan generar nuevas formas de trabajar y transformar la práctica pedagógica. No es tarea fácil, toda vez que existe gran diversidad cultural, creencias, necesidades, intereses,personalidades y conductas de todos tipos. En este contexto, el desempeño docente tiene lugar cuando no solo es necesario contar con conocimiento, sino que es necesario poseer las HHSS, tener convicción y disposición para aplicarlas.Recordando a Delors en 1996 con su informe en la UNESCO donde nos dio a conocer los cuatro pilares de la educación, saber, hacer, ser y convivir, vale la pena reflexionar si estas bases teóricas han logrado su propósito en la experiencia real de los docentes en primera instancia.

Pensando en el docente como profesional en actitud de aprendizaje y en su rol como agente de cambio y transformación social, coincido con Garza y Leventhal (1998) que refieren que el proceso de aprendizaje no solamente 
consiste en adquirir conocimiento sino también en el desarrollode habilidades que influyan en la personalidad; en la autonomía, en la actitud positiva; en la autorregulación, en la responsabilidad de los propios resultados de aprendizaje y el actuar en concordancia con esto. En este sentido, la competencia social del profesor como actor en el escenario educativo es de importancia trascendente. Como autora del presente estudio concuerdo con López e Iriarte (2004) que mencionaron: "El concepto de competencia social en la educación engloba las perspectivas educativas más modernas relativas al ámbito de la integración social y prevención del fracaso personal y social".

Por lo anterior expuesto, el presente trabajo tiene como objeto de estudio las HHSS del docente de EB como un primer escalón para una transformación con la intención de contribuir a un cambio que finalmente impacte en la práctica educativa. Se establece esta misma problemática a manera de pregunta de la siguiente manera: ¿Cómo inciden las HHSS del docente en la promoción de nuevas formas de saber, hacer y ser desde la visión de los actores de EB?

Se establece un diseño de investigación con estrategia de estudio de caso, enfoque cualitativo y de alcance descriptivo.

\section{Justificación}

La importancia de realizar estudios como el presente radica principalmente en que no existe suficiente información científica relacionada. Por otra parte, cuando hablamos delos referentes involucrados en la calidad de la educación es necesario primeramente observar de qué manera se están involucrandoestos conceptos teóricos en el quehacer pedagógico. Por lo anterior, se pretende observar la función de las HHSS en el trabajo de los docentes para describir su impacto en la experiencia educativa.

Los resultados de esta investigación beneficiarán a los docentes al brindarles evidencia de aspectos en su desempeño que probablemente no hayan observado y que les permitan retroalimentar su práctica y mejorar, en segunda instancia a los alumnos por contar con una instrucción más eficaz, y en general a la comunidad escolar al contar con más recursos para alcanzar la calidad educativa planteada en los planes y programas de estudio. Así mismo, al revelar algunos aspectos de la manera en que se aplican las bases teóricas de las HHSS en la práctica estaremos en posibilidad de diseñare implementar programas de entrenamiento que faciliten el logro de metas planteadas por los organismos internacionales y nacionales en favor de los procesos de enseñanza aprendizaje.Finalmente, la 
sociedad se beneficia indirectamente desde el punto de vista en que los egresados de EB cuenten con un perfil de egreso capaz de responder a demandas sociales propias de su entorno.

\section{Objetivo general}

Analizar el desarrollo y construcción de doshabilidades sociales en un colectivo de docentes de EducaciónBásica, mediante un seguimiento a sus actividades cotidianas en el aula para indagar la manera en que se promueven y determinar áreas de oportunidad.

\section{Objetivo específico}

Revelar las formas oroles de trabajoque tienen docentes con referencia al uso y desarrollo de las dos Habilidades Sociales: Asertividad e Inteligencia Emocional.

\section{Preguntas de investigación}

- ¿Qué roles de trabajoadoptan los docentes al aplicar las bases teóricas de las dosHHSS en los procesos de enseñanza aprendizaje?

- ¿Qué estrategias de trabajose utilizan para promoverel desarrollo de las dos HHSS?

- ¿Qué áreas de oportunidad existen para el diseño e implementación de programas de entrenamiento en posteriores estudios?

\section{Fundamentos teóricos}

\section{Concepto de habilidades sociales}

Varios expertos afirman que es imposible desarrollar una definición consistente de competencia social puesto que ésta es parcialmente dependiente del contexto cambiante.Se considera que las habilidades sociales permiten a una persona funcionar en su contexto. Anaya, en el año 1991, manifiesta que la habilidad social es la capacidad de actuar coherentemente con el rol que los demás esperan de nosotros.

Caballo (1993) define las habilidades sociales como un conjunto de conductas emitidas por el individuo en un contexto interpersonal que expresa sus sentimientos, actitudes, deseos, opiniones o derechos de un modo adecuado a la situación. Dos conceptos concretos son: "La capacidad de expresar interpersonalmente sentimientos positivos ynegativos sin que dé como resultado una pérdida de reforzamiento social" y "La expresión adecuada, dirigida hacia otra persona, de cualquier emoción que no sea la respuesta de ansiedad". 
RECIE. Revista Electrónica Científica de Investigación Educativa Vol. 4, núm. 2, enero-diciembre 2019, pp. 1303-1315.

\section{Asertividad}

Es una habilidad social que implica a formarse a sí mismo, es comunicar nuestras creencias, sentimientos y opiniones de manera directa, honesta, adecuada, oportuna, considerada y respetuosa. Es la habilidad para emitir conductas que afirmen o ratifiquen la propia opinión, sin emplear conductas agresivas para los demás. Es un comportamiento de expresión directa de los propios sentimientos y de defensa de los derechos personales. Sirve para ser es capaz de expresar sentimientos o deseos positivos y negativos de forma eficaz sin sentir vergüenza, discriminar entre la aserción, agresión y pasividad, defenderse sin agresión o pasividad frente a la conducta poco cooperadora o razonable de los demás, defender una postura personal frente a una situación o tema sin dejarse presionar para cambiarla.

\section{Inteligencia emocional}

Daniel Goleman (1995) la define como un conjunto de capacidades, competencias y habilidades no cognitivas que influyen en la habilidad propia de tener éxito al afrontar las demandas y presiones del medio ambiente y que implican emociones. Sus componentes son autoconocimiento emocional, autocontrol emocional o autorregulación, automotivación, reconocimiento de emociones ajenas o empatía, relaciones interpersonales o habilidades sociales, mantener buenas relaciones con los demás va a resultar fundamental en nuestras vidas, en cualquier contexto en el que nos desenvolvamos. Esto va a implicar saber cómo comportarse en diferentes situaciones. En palabras del mismo autor, una educación o alfabetización emocional posibilitaa las personas controlar sus emociones.

\section{Teoría de las representaciones sociales}

Materán (2008) menciona que no son simples opiniones, sino que son construcciones simbólicas que se crean y recrean en el curso de las interacciones sociales; son maneras específicas de entender y comunicar la realidad. Su finalidad es la de transformar lo desconocido en algo familiar, son como conjuntos estructurados o imprecisos de nociones, creencias, imágenes, metáforas y actitudes con los que los actores definen las situaciones y llevan a cabo sus planes de acción. En el ámbito de la educación, el modo en que las representaciones sociales intervienen en las prácticas educativas constituye un objeto pertinente para la investigación, porque participan en la formación de los conocimientos escolares ya que no es un asunto meramente cognoscitivo. 


\section{Paradigma de las creencias pedagógicas}

Según Rojas (2014) este paradigma está representado por un cúmulo de investigaciones provenientes de la pedagogía, la psicología y también la etnografía que giran en torno a la importancia del actor docente en los procesos de interacción y socialización en el aula. Esta línea de estudios considera que existe un conocimiento propio de los docentes que se adquiere y se utiliza de forma activa en su práctica profesional. Este conocimiento se refiere a saberes, convicciones, creencias, actitudes, reflexiones, expectativas, opiniones, explicaciones, entre otras, que se movilizan en la práctica docente de forma automática o reflexiva. Las creencias son "un conjunto de potentes esquemas sobre la educación, el conocimiento, el aprendizaje y la enseñanza, a partir de las cuales los docentes toman decisiones conscientes y priorizadas". También se las consigna como juicios personales que predicen formas de comportamiento y que guían los pensamientos de las personas. Estas se organizan en base a "las conexiones y relaciones con otras creencias $\mathrm{u}$ otras estructuras cognitivas o afectivas; cumplen una función adaptativa fundamental.

\section{Método}

El estudio se realiza en los municipios de Camargo y Saucillo, del estado de Chihuahua, en dos escuelas preescolares, tres primarias una de ellas rural y un Centro de Atención Múltiple (CAM). La población del presente estudio está representada por docentes, directores y practicantes.

El diseño de investigación consiste en un estudio de caso, enfoque cualitativo, con alcance descriptivo.Las técnicas que se utilizan fueron guía para entrevista y notas de diarios de campo utilizados por los practicantes. El procedimiento se iniciócon la inmersión en el campo compartiendo el recreo con algunos maestros y platicando a cerca de varias situaciones escolares propias del proceso de enseñanza aprendizaje, posteriormente la inmersión total consistió en abordar la manera en que trabajan las habilidades sociales en el aula, y abordando también a los directores y en tercera instancia a los practicantes en cada una de las escuelas.Se les aplicó una entrevista primero a los docentes luego a los directores y después a los practicantes, los docentes fueron entrevistados algunos en sus salones de clases y otros en los patios de sus escuelas, todas las entrevistas se realizaron en la hora del recreo o después de clases. Las notas de los diarios de campo fueron elaboradas por los practicantes en sus sesiones de prácticas por medio de la observación durante el trabajo en las aulas.El análisis de los datos inició con la captura de los mismos en tablas de 
distribuciónde frecuencias para cada una de las preguntas en el programa de Excel versión 2016.

\section{Análisis de los datos}

Pregunta 1. ¿Considera que los docentes cuentan suficientemente con las siguientes HHSS para formarlas en los alumnos conforme a los planes y programas para la EB?

- Inteligencia emocional

- Asertividad

Si no ¿Porqué?

\section{Tabla 1}

Frecuencias de respuesta pregunta 1

\begin{tabular}{|c|c|c|c|}
\hline Respuestas & docentes & directores & practicantes \\
\hline SI & 6 & 3 & 9 \\
\hline NO & 8 & 4 & 14 \\
\hline $\begin{array}{c}\text { No } \\
\text { contesto }\end{array}$ & 2 & & \\
\hline
\end{tabular}

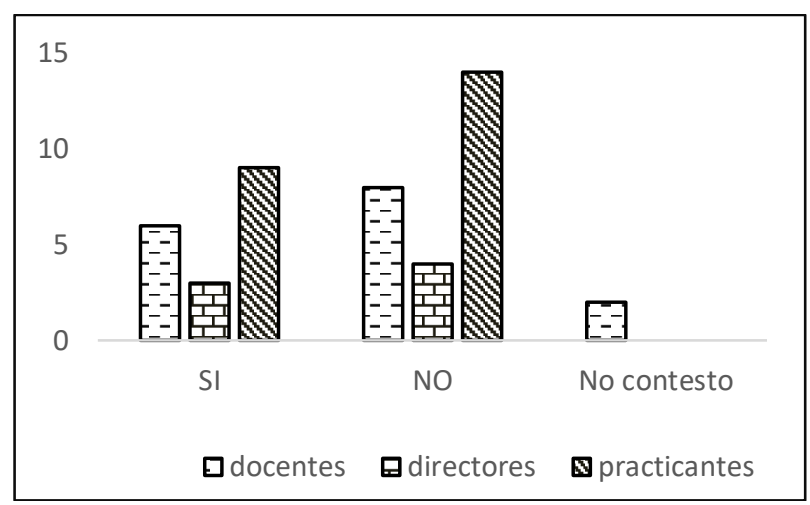

Figura 2. Respuestas pregunta 1.

Más de la mitad (57\%) de los participantes contestó que los docentes no cuentan con las HS suficientes, el (40 \%) de los participantes contestó que sí. Pregunta 2. ¿Considera que las creencias pedagógicas influyen en la manera de impartir las HS en el aula?

si no ¿Porque? 
Tabla 2

Frecuencia de respuestas pregunta 2

\begin{tabular}{|l|r|r|r|}
\hline Respuestas & docentes & directores & practicantes \\
\hline SI & 12 & 6 & 17 \\
\hline NO & 1 & 1 & 3 \\
\hline $\begin{array}{l}\text { No } \\
\text { contesto }\end{array}$ & 3 & & 3 \\
\hline
\end{tabular}

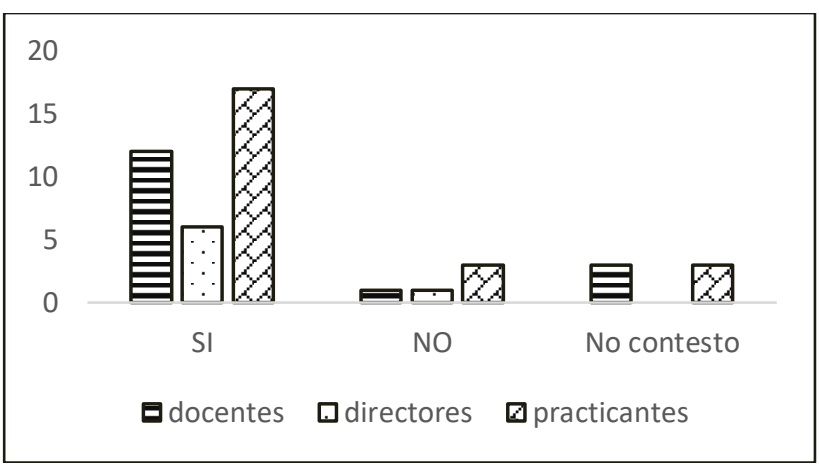

Figura 3. Respuestas pregunta 2

La mayoría (76\%) contesto que sí. Una minoría (11\%), contesto que no. Pregunta 3. ¿Qué recomienda para que los docentes desarrollen las HHSS ante esta problemática?

Una de las recomendaciones que más se repitió fue la capacitación presencial o mixta, otra de las respuestas más frecuentes fue la apertura, el estar abiertos al cambio y no tener miedo a cambiar las antiguas formas de trabajo, el dejar antiguos paradigmas y ser mas humanistas y con más sensibilidad, otras recomendaciones fueron conocer más de las HHSS para poder comprenderlas mejor y compartir estrategias con otros docentes para poder retroalimentarse mejor.

Pregunta 4. ¿Cree que ayudaría un taller para trabajar Creencias Pedagógicas?

Tabla 3

Frecuencia de respuestas pregunta 4

\begin{tabular}{|l|r|r|r|}
\hline Respuestas & docentes & directores & practicantes \\
\hline SI & 14 & 7 & 23 \\
\hline NO & & 0 & 0 \\
\hline $\begin{array}{l}\text { No está } \\
\text { seguro }\end{array}$ & 2 & & \\
\hline
\end{tabular}

Habilidades sociales en la práctica docente: una mirada desde los actores... 
RECIE. Revista Electrónica Científica de Investigación Educativa

Vol. 4, núm. 2, enero-diciembre 2019, pp. 1303-1315.

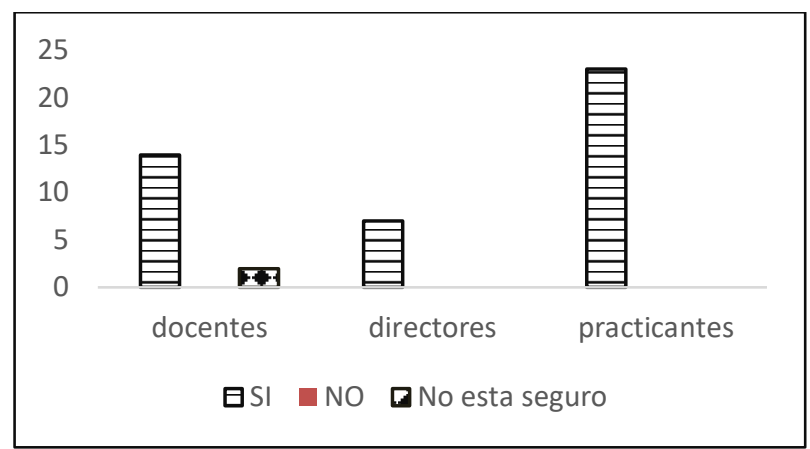

Figura 4. Respuestas figura 4

Casi el total de los participantes (96\%) contesto que sí.

\section{Conclusión}

De acuerdo a los resultados, existe una inquietud entre los docentes participantes respecto a que no cuentan con las HS suficientes, expresaron que falta más capacitación y que las actualizaciones que reciben no han cumplido sus expectativas ya que todas son virtuales y necesitan que sean presenciales o mixtas. Hablaron de un malestar docente que impide la tolerancia y la empatía con los niños, y no favorece la actitud asertiva.

Un grupo considerable de participantes refirieron que los docentes tienden a "casarse" con una teoría pedagógica y es muy difícil cambiarla, mencionan que influye la ideología, sus patrones pedagógicos aprendidos desde hace muchos años, la formaciónbrindada por sus instituciones de procedencia, etc. Si bien se dejó establecido que los planes y programas se estudian, aun así, es difícil combinar las teorías pedagógicas pasadas con las de nuevos modelos, un hallazgo importante es que precisaron la necesidad de capacitación en cómo hacer esta transición, así mismo demandan entrenamiento en la apertura al cambio hacia nuevas formas de trabajo y dejar antiguos paradigmas.

Las debilidades en las dos HHSS estudiadas fueron evidenciadas ya que los participantes reconocen que,para alcanzar un dominio en ellas, es necesario ser más humanistas y para tener empatía se requiere más sensibilidad. En este punto solicitan apoyo para conocer más de las HHSS, para estar en posibilidad de comprenderlas mejor, cambiar paradigmas y cambio de patrones de creencias a cerca de la pedagogía con la que trabaja.

Sin duda el lograr que los profesores conozcan sus fortalezas o sus debilidades en cuanto sus propias HHSS es un reto muy grande, fue muy interesante escuchar las necesidades de capacitación que demandan los colectivos de docentes y su sentir respecto a la manera en que el sistema 
educativo les está brindando los cursos en línea de actualización y como les está funcionando.

Uno de los principales hallazgos es la necesidad de trabajar las creencias pedagógicas de los docentes.Otro importante hallazgo es la demanda que existe por parte de los docentes de que se les capacite para combinar lo esencial de las teorías pedagógicas de los antiguos modelos educativos con las nuevas tendencias, toda vez que algunos participantes manifiestan no estarrenuentes al cambio, sino que necesitan aprender cómo hacerlo.

Se deja evidencia del interés de los actores de la comunidad escolar en cursos o talleres con estas temáticas para conocer mejor sus HHSS, comprenderlas y aplicarlas de mejor manera, así mismo refieren que para alcanzar el ideal del rol docente como educador social capaz de generar un cambio es necesario más sensibilización ante esta realidad social, talleres con esta ultima necesidad también son requeridos por directores y docentes, en virtud de que casi el total expresó que uno de los más grandes obstáculos para la correcta aplicación de las HHSS en el aula es la falta apertura al cambio.

Es alentador ver la disposición hacia la actualización de los participantes, sin embargo, es necesario atender sus demandas, y en el caso de dos escuelas rurales se encontró que sus condiciones de infraestructura no facilitan el acceso a la actualización del sistema educativo en modalidad virtual.

La evidencia recogida por la presente investigación confirma los conceptos de creencias pedagógicas al estilo de Rojas en 2014 cuando se refiere al profesor con poderosos esquemas que dirigen sus decisiones educativas. El interés que despertó en los directores participantes la expectativa de un taller de creencias pedagógicas deja a la autora de este estudio con un compromiso aun más grande de seguir estudiando el caso de las HHSS y la forma del quehacer pedagógico en torno a ellas, sin perder de vista las nuevas tendencias de la reforma educativa vigente, así mismo se deja precedente para seguir investigando este hecho, aplicando mas estrategias de investigación para un mayor y mejor acercamiento a la problemática planteada.

Con base en los hallazgos y por lo anterior expuesto, una importante recomendación es el diseño de un taller de creencias pedagógicas y las HHSS (asertividad e inteligencia emocional) elaborado por especialistas en la materia con reconocida experiencia y con amplios conocimientos en el humanismo y la pedagogía, dirigido a docentes e impartido en modalidad presencial. Es esencial que el enfoque humanista se aplique de manera 
RECIE. Revista Electrónica Científica de Investigación Educativa Vol. 4, núm. 2, enero-diciembre 2019, pp. 1303-1315.

transversal, se realice un trabajo de sensibilización, así mismo que la inclusión, la equidad y la empatía permeen en todas las actividades que se incluyan. La tolerancia y el dialogo deberán también estar presentes y ser parte nodal de este taller para que llegue a un buen término y se alcancen los propósitos educativos de calidad del trabajo docente.

\section{Referencias}

Acosta, M. (2009). Acercamiento a los planteamientosde Donald Schön.

Igualdad social. XXVII Congreso de la Asociación Latinoamericana de Sociología. VIII Jornadas de Sociología de laUniversidad de Buenos Aires. Asociación Latinoamericana deSociología, Buenos Aires.

Recuperado de: https://www.aacademica.org/000-062/1964

Anaya, J. (1991). Recursos psicotécnicos y bibliográficos para equipos interdisciplinares. Madrid: M.E.C., D.G.R.P. Recuperado de: https://www.mipsicologovirtual.com/wptent/uploads/2013/05/habilidades-sociales.doc

Caballo, V. (1993). Manual de evaluación y entrenamiento de las habilidades sociales. Siglo XXI. España.

Flick, U. (2007). Introducción a la Investigación Cualitativa. 2ª Ed. Madrid. Ediciones Morata.

García, A. (2010). Estudio sobre la asertividad y las habilidades sociales en el alumnado de educación social. Recuperado de: http://rabida.uhu.es/dspace/bitstream/handle/10272/5352/b1645 0188.pdf?sequence $=2$

Garza, R.\&Leventhal, S. (2000).Aprender cómoaprender. Mexico: Trillas. Recuperado de http://www.ljemail.org/reference/ReferencesPapers.aspx?Referenc $\underline{\mathrm{eID}=1408346}$

Gimeno, J. (1998). Poderes inestables en educación. Madrid. Ediciones Morata.

Gimeno, J., Pérez, A., Martínez, J., Torres, J., Angulo, F. y Álvarez, J. (2008). Educar por competencias, ¿qué hay de nuevo? Madrid. Ediciones Morata.

Goleman, D. (1995). Emotionalintelligence. Nueva York: Bantam Books.

González Quiñones César Augusto y Pinedo Valladares Fiorella. (s.f.). Habilidades sociales. Universidad Peruana de los Andes. Recuperado de: $\quad$ http://www.monografias.com/trabajos96/las-habilidadessociales/las-habilidades-sociales2.shtml\#ixzz5C1BQZAai 
Hernández, S., Fernández. y Baptista, P. (2003). Metodología de la Investigación. McGraw-Hill. México.

López, N., Iriarte, C., González, M. (2004). Aproximación y revisión del concepto «competencia social». Universidad de navarra. Recuperado de: https://dialnet.unirioja.es/descarga/articulo/866882.pdf

Materán, Angie Las representaciones sociales: un referente teórico para la investigación educativa Geoenseñanza, vol. 13, núm. 2, juliodiciembre, 2008, pp. 243-248 Universidad de los Andes San Cristobal, Venezuela.

Rojas, M. Facultad de Educación, Universidad Alberto Hurtado. Santiago, Chile. Las creencias docentes: delimitación del concepto y propuesta para la investigación. Revista electrónica diálogos educativos issn: 0718-1310 № 27 Vol. 14 - año 2014. Recuperado de:http://www.dialogoseducativos.cl/revistas/n27/rojas

Secretaria de Educación Pública (2017). Aprendizajes Clave para la educación Integral. Plan y programas de estudio para la educación básica. México.

Recuperado de:http://www.aprendizajesclave.sep.gob.mx/

UNESCO. (1999). Declaración sobre una cultura de la paz. Recuperado de: www.unesco.org 
RECIE. Revista Electrónica Científica de Investigación Educativa Vol. 4, núm. 2, enero-diciembre 2019, pp. 1303-1315. 